\title{
Evaluation of SHP1-P2 methylation as a biomarker of lymph node metastasis in patients with squamous cell carcinoma of the head and neck
}

\author{
Nakarin Kitkumthorn ${ }^{1, *}$, Somboon Keelawat ${ }^{2}$, Jutamas Wongphoom³, Prakasit Rattanatanyong ${ }^{2, t}$, \\ Apiwat Mutirangura²
}

\begin{abstract}
Background: Hypermethylation of $S r c$ homology region 2 domain-containing protein-tyrosine phosphatase 1 promoter 2 (SHP1-P2) has been proven as an epithelial-specific marker. This marker has been used for the detection of lymph node metastasis in patients with lung cancer or colon cancer.

Objectives: To investigate SHP1-P2 methylation in patients with squamous cell carcinoma of the head and neck (HNSCC) and determine its potential for micrometastasis detection in the lymph nodes of patients with HNSCC.

Methods: SHP1-P2 methylation levels were analyzed by combined methylation-specific primer TaqMan real-time PCR in 5 sample groups: normal tonsils $(n=10)$, microdissected squamous cell carcinoma epithelia $(n=9)$, nonmetastatic head and neck cancer lymph nodes ( $\mathrm{LN} \mathrm{N0}, \mathrm{n}=15$ ), metastatic HNSCC histologically negative for tumor cells (LN-, $\mathrm{n}=18)$, and matched cases histologically positive for tumor cells $(\mathrm{LN}+, \mathrm{n}=18)$.

Results: SHP1-P2 methylation of $10.27 \pm 4.05 \%$ was found in normal tonsils as a lymphoid tissue baseline, whereas it was $61.31 \pm 17.00 \%$ in microdissected cancer cell controls. In the 3 lymph node groups, the SHP1-P2 methylation levels were $9.99 \pm 6.61 \%$ for LN N0, $14.49 \pm 10.03 \%$ for LN- Nx, and $41.01 \pm 24.51 \%$ for LN+ Nx. The methylation levels for $\mathrm{LN}-\mathrm{Nx}$ and $\mathrm{LN}+\mathrm{Nx}$ were significantly different $(P=0.0002)$. Receiver operating characteristic curve analysis of SHP1-P2 methylation demonstrated an area under the curve of 0.637 in distinguishing LN N0 from LN-Nx. Conclusions: SHP1-P2 methylation was high in HNSCC, and low in lymphoid tissues. This methylation difference is concordant with lymph node metastasis.
\end{abstract}

Keywords: DNA methylation; lymph nodes; neoplasm micrometastasis; promoter regions, genetic; squamous cell carcinoma of head and neck; Src homology region 2 domain-containing protein-tyrosine phosphatase 1

\footnotetext{
*Correspondence to: Nakarin Kitkumthorn, Department of Oral Biology, Faculty of Dentistry, Mahidol University, Bangkok 10400, Thailand, e-mail: nakarin.kit@mahidol.ac.th;

†Prakasit Rattanatanyong, deceased January 2, 2017.

'Department of Oral Biology, Faculty of Dentistry, Mahidol University, Bangkok 10400, Thailand ${ }^{2}$ Department of Pathology, Faculty of Medicine, Chulalongkorn University, Bangkok 10330, Thailand

${ }^{3}$ Department of Pathology, King Chulalongkorn Memorial Hospital, Thai Red Cross Society, Bangkok 10330, Thailand ${ }^{4}$ Center of Excellence in Molecular Genetics of Cancer and Human Diseases, Department of Anatomy, Faculty of Medicine, Chulalongkorn University, Bangkok 10330, Thailand ¿ Open Access. ( 2018 Nakarin Kitkumthorn et al., published by Sciendo. (c) Br-Nc-ND This work is licensed under the Creative Commons Attribution NonCommercial-NoDerivatives 4.0 License.
} 
Squamous cell carcinoma of the head and neck (HNSCC) is a common form of cancer with high mortality, and the number of cases is increasing annually [1]. HNSCC can be fatal, frequently from metastasis. In cases of single unilateral lymph node metastasis, the 5-year survival rate is less than $50 \%$, and in cases of bilateral metastases, the survival is less than $25 \%$ [2]. In general, histological biopsy is the criterion standard for HNSCC metastasis detection in lymph nodes, but this is uncomfortable for patients and requires an experienced pathologist for assessment. Moreover, limitations, such as inadequate tissue sampling and oversights in detecting minute tumor cells, make identification of metastatic HNSCC difficult. This may lead to underdiagnosis and inaccurate tumor staging, resulting in inappropriate therapeutic management [3-7]. Thus, a biomarker to detect metastatic HNSCC and replace the traditional histological method is highly desirable. The $S r c$ homology region 2 domain-containing proteintyrosine phosphatase 1 promoter 2 (SHP1-P2) is specifically methylated in epithelial tissue and unmethylated in other tissues, including blood vessels, nerves, fibroblasts, and white blood cells [8-10]. In addition, methylated SHP1-P2 DNA has been observed in cancer cells originating from epithelial cells $[9,11,12]$. Hence, if methylated SHP1-P2 DNA is present, it is likely to be derived from metastasized epithelial cancer cells.

Recently, our group reported the presence of methylated SHP1-P2 DNA in the plasma and lymph nodes of patients with nonsmall-cell lung cancer, whereas methylated SHP1-P2 DNA was absent in cancer-free individuals $[9,11]$. Later, we developed an efficient method for the measurement of SHP1-P2 methylation, we called combined methylation-specific primer TaqMan real-time (COMST) polymerase chain reaction (PCR) [12]. This method can detect colorectal cancer (CRC) DNA in lymph nodes even if cancer cells are not visible under a microscope, suggesting its potential for detecting markers of epithelial cancer metastasis in lymph nodes.

In the present study, we aimed to determine whether measurement of SHP1-P2 methylation can be used as an effective biomarker to detect metastatic HNSCC and whether these measurements can provide support for diagnosing nodal metastases from HNSCC as a possible alternative to routine histopathology.

\section{Materials and methods}

\section{Sample inclusion}

The present study was approved by the Institutional Review Board (IRB) of the Faculty of Medicine, Chulalongkorn
University, Thailand (IRB No. 516/56, approval No. 004/2014). All samples were coded-anonymized formalinfixed, paraffin-embedded tissue retrieved retrospectively from the Department of Pathology, Faculty of Medicine, Chulalongkorn University, affiliated with King Chulalongkorn Memorial Hospital, a tertiary care, university teaching hospital in central Bangkok, Thailand. The samples included 10 normal tonsils, and samples from 51 patients diagnosed with HNSCC including 9 samples of primary HNSCC and 42 lymph nodes from patients with and without HNSCC metastasis as inclusion criteria. With the exception of one 11-year-old boy, all other patients were in their fourth decade or older at the time of clinical sampling. Lymph nodes had been derived from radical neck dissections and were classified into 3 types: (1) lymph nodes from patients with control cases of nonmetastatic HNSCC (LN N0, n = 15), (2) lymph nodes from patients with cases of metastatic HNSCC, but histologically negative for carcinoma cells $(\mathrm{LN}-\mathrm{Nx}, \mathrm{n}=18)$, and (3) lymph nodes from patients with matched cases of metastatic HNSCC that were histologically positive for carcinoma cells $(\mathrm{LN}+\mathrm{Nx}, \mathrm{n}=18)$. The normal tonsils and the LN N0 and LN- Nx types were confirmed histologically to be free of carcinoma cells, whereas lymph nodes of the $\mathrm{LN}+\mathrm{Nx}$ type were diagnosed as having metastatic malignant cells in at least $70 \%$ of the nodal tissue. All samples were confirmed as meeting the criteria by consensus from 2 pathologists (NK and SK). The demographic data of the patients, clinical stages, and histological grades were reviewed from their medical records and are presented in Table 1.

\section{DNA extraction and bisulfite modification}

All formalin-fixed, paraffin-embedded (FFPE) tissues were sliced into 3 to 5 sections of $5 \mu \mathrm{m}$ thickness and left unstained. Another section was stained with hematoxylin and eosin (HE) for histopathological confirmation. In the HNSCC groups, tumor cells were manually microdissected as previously reported [13]. In brief, FFPE tissues were cut serially into 5 levels. The first and the last of the 5 slides were stained with HE. Selected areas on the first slide were circled using a marker pen. Subsequently selected areas on the last slide were also marked using the first slide as a reference and subsequently examined under a microscope. If the last slide was correctly marked, the remaining unstained slides (levels 2-4) would be processed in the same manner using the first and last HE-stained slides as references to select the areas. Later, the marked areas on the unstained slides were dissected using a standard 21-gauge needle. After deparaffinization with xylene, the DNA was isolated using Tris-buffered sodium 
Table 1. Demographic data of patients with squamous cell carcinoma of the head and neck (HNSCC)

\begin{tabular}{lclcc}
\hline Sex & Age (y) & Organ & $\begin{array}{c}\text { Histological } \\
\text { grade }\end{array}$ & $\begin{array}{c}\text { Clinical } \\
\text { stage }\end{array}$ \\
\hline \multicolumn{4}{l}{ Microdissected } & HNSCC \\
M & 51 & Floor of mouth & 2 & 2 \\
F & 62 & Tongue & 1 & 1 \\
M & 65 & Floor of mouth & 2 & 3 \\
F & 62 & Tongue & 3 & 1 \\
M & 67 & Gingiva & 1 & 2 \\
M & 50 & Gingiva & 1 & 2 \\
M & 61 & Tongue & 2 & 4 \\
M & 37 & Tongue & 2 & 2 \\
M & 56 & Tongue & 3 & 4 \\
\hline
\end{tabular}

\begin{tabular}{lllll}
\hline No (no metastasis) & & 2 & \\
11 & $\mathrm{M}$ & Tongue & 1 & 4 \\
67 & $\mathrm{~F}$ & Buccal mucosa & 1 & 3 \\
47 & $\mathrm{~F}$ & Tongue & 1 & 2 \\
53 & $\mathrm{~F}$ & Tongue & 2 & 1 \\
51 & $\mathrm{M}$ & Floor of mouth & 2 & 3 \\
83 & $\mathrm{~F}$ & Lip & 1 & 4 \\
70 & $\mathrm{M}$ & Soft palate & 1 & 3 \\
48 & $\mathrm{~F}$ & Soft palate & 2 & 4 \\
72 & $\mathrm{M}$ & Palate & 2 & 2 \\
38 & $\mathrm{~F}$ & Tongue & 1 & 2 \\
46 & $\mathrm{~F}$ & Tongue & 2 & 1 \\
65 & $\mathrm{M}$ & Buccal mucosa & 2 & 3 \\
81 & $\mathrm{M}$ & Tongue & 2 & 1 \\
72 & $\mathrm{~F}$ & Buccal mucosa & 2 & 2 \\
63 & $\mathrm{M}$ & Tongue & & 1 \\
\hline
\end{tabular}

\section{Nx (metastasis)}

\begin{tabular}{lllll}
52 & M & Tongue & 1 & 1 \\
57 & M & Soft palate & 3 & 3 \\
54 & M & Soft palate & 2 & 4 \\
56 & M & Tongue & 2 & 4 \\
79 & F & Palate & 3 & 4 \\
74 & M & Buccal mucosa & 1 & 4 \\
42 & M & Tongue & 3 & 4 \\
66 & M & Soft palate & 2 & 4 \\
51 & M & Tongue & 2 & 4 \\
66 & F & Buccal mucosa & 1 & 2 \\
58 & M & Tongue & 2 & 4 \\
67 & M & Tongue & 2 & 4 \\
60 & M & Buccal mucosa & 1 & 4 \\
69 & M & Gingiva & 2 & 4 \\
34 & M & Tongue & 1 & 4 \\
64 & F & Tongue & 2 & 3 \\
61 & $M$ & Soft palate & 2 & 3 \\
84 & F & Lower lip & 2 & 4 \\
\hline
\end{tabular}

Histological grade: 1 = well-differentiated, 2 = moderately-differentiated, 3 = poorly-differentiated

dodecyl sulfate with proteinase $\mathrm{K}$ and then left at $50^{\circ} \mathrm{C}$ overnight, followed by phenol-chloroform extraction and ethanol precipitation. The isolated genomic DNA was eluted and then used for bisulfite treatment. Bisulfite modification of the genomic DNA was performed using an EZ DNA Methylation Kit (Zymo Research). The bisulfite-treated DNA was resuspended in $20 \mu \mathrm{L}$ of water and stored at $-20^{\circ} \mathrm{C}$ until needed for use.

\section{COMST PCR}

Quantification of SHP1-P2 methylation levels was performed using a combination of methylation-specific primers and the absolute quantitative TaqMan probe real-time PCR technique, as previously published [12]. Briefly, the methylated and unmethylated sets were used to quantitate the amount of methylated and unmethylated DNA, respectively. The methylation set contained 5'-AGGATTTATT CGATGATAGTTGTTATCGTT-3' as the forward primer, 5'-CTCCACCAACTACTTTTACGCAAC-3' as the reverse primer, and 5'-FAM-CTAACCCACGCTAATAA-MGB-3' as the probe. The unmethylated set comprised 5'-GTAGGATT TATTTGATGATAGTTGTTATTG- $3^{\prime}$ as the forward primer, 5'-TCCTCCACCAACTACTTTTACACAA- $3^{\prime}$ as the reverse primer, and 5'-VIC-CCCTAACCCACACTA-MGB-3' as the probe. The 2 PCR sets were normalized with the $\beta$-actin control set, which was composed of the forward primer 5'-GTGTATTTGATTTTTGAGGAGA-3', the reverse primer 5'-CCTTAATACCAACCTACCCAA-3', and the probe 5'-Cy5AAGGTGAAYGTGGATGAAGTTGGTGGTGAGGBHQ-3'.

All PCRs were performed as duplex PCR using $2 \times$ TaqMan GTXpress Real-Time PCR Master Mix (Applied Biosystems) with an ABI 7500 fast real-time PCR system. The SHP1-P2 methylation level was calculated using the following equation:

$$
\begin{gathered}
\text { Percentage of SHP1-P2 methylation } \\
=(\mathrm{nM} \times 100) /(\mathrm{nM}+\mathrm{nU})
\end{gathered}
$$

where $\mathrm{nM}$ (normalized methylated DNA) and nU (normalized unmethylated DNA) are the levels of methylated DNA or unmethylated DNA, respectively, divided by the level of $\beta$-actin in the same reaction. The copy numbers for methylated DNA, unmethylated DNA, and $\beta$-actin were obtained by comparing the change in the fluorescence signal $(\Delta \mathrm{RN})$ of the target DNA minus the RN from a passive reference dye for a given reaction of each target with a standard curve generated using varying concentrations of each target. To determine whether SHP1-P2 methylation is epithelial carcinoma cell specific, tonsil tissue and microdissected HNSCC epithelia were used as negative and positive controls, respectively. 


\section{Statistical analyses}

All statistical analyses were conducted using IBM SPSS Statistics for Windows (version 22). Descriptive data for the SHP1-P2 methylation levels are expressed as mean \pm standard deviation. Independent sample Student $t$ tests were conducted to determine the differences between all groups except for $\mathrm{LN}-\mathrm{Nx}$ and $\mathrm{LN}+\mathrm{Nx}$, for which a paired Student $t$ test was applied. $P<0.05$ was considered as significant. A receiver operating characteristic curve analysis was conducted to assess the ability of the SHP1-P2 methylation level to differentiate between lymph nodes with or without metastases.

\section{Results}

\section{Comparison of SHP1-P2 methylation levels in tonsils and microdissected HNSCC epithelia}

The level of SHP1-P2 methylation from the tonsil samples was $10.27 \pm 4.05 \%(n=10)$ compared with $61.31 \pm 17.00 \%$ $(\mathrm{n}=9)$ in primary microdissected HNSCC epithelia (Figure 1A).

\section{SHP1-P2 methylation level in the 3 lymph node groups}

The SHP1-P2 methylation in LN N0, LN- Nx, and LN+ Nx was $10.27 \pm 4.05 \%, 14.49 \pm 10.03 \%$, and $41.01 \pm 24.51 \%$, respectively. A significant difference was observed between LN N0 and LN+ Nx. In addition, the paired $t$ test showed a significant difference $(P<0.0001)$ between LN- Nx and LN+ $\mathrm{Nx}$ (Figure 1A and $\mathbf{B}$ and Table 2). There were no significant differences in SHP1-P2 methylation levels between different sex, ages, stages, and tumor grades (data not shown).

\section{Evaluation of SHP1-P2 methylation level to detect}

\section{HNSCC micrometastases in lymph nodes}

Comparing the SHP1-P2 methylation levels for LN N0 and LN- Nx, the percent SHP1-P2 methylation yielded a maximal area under the curve at 0.637 . This value could be used detect occult carcinomas with a cutoff value of $10.14 \%$, sensitivity of $66.67 \%$, and specificity of $60 \%$ (Figure 1C).

\section{Discussion}

Metastatic HNSCC has a high mortality and a low cure rate [14]. Various methods have been developed for detecting
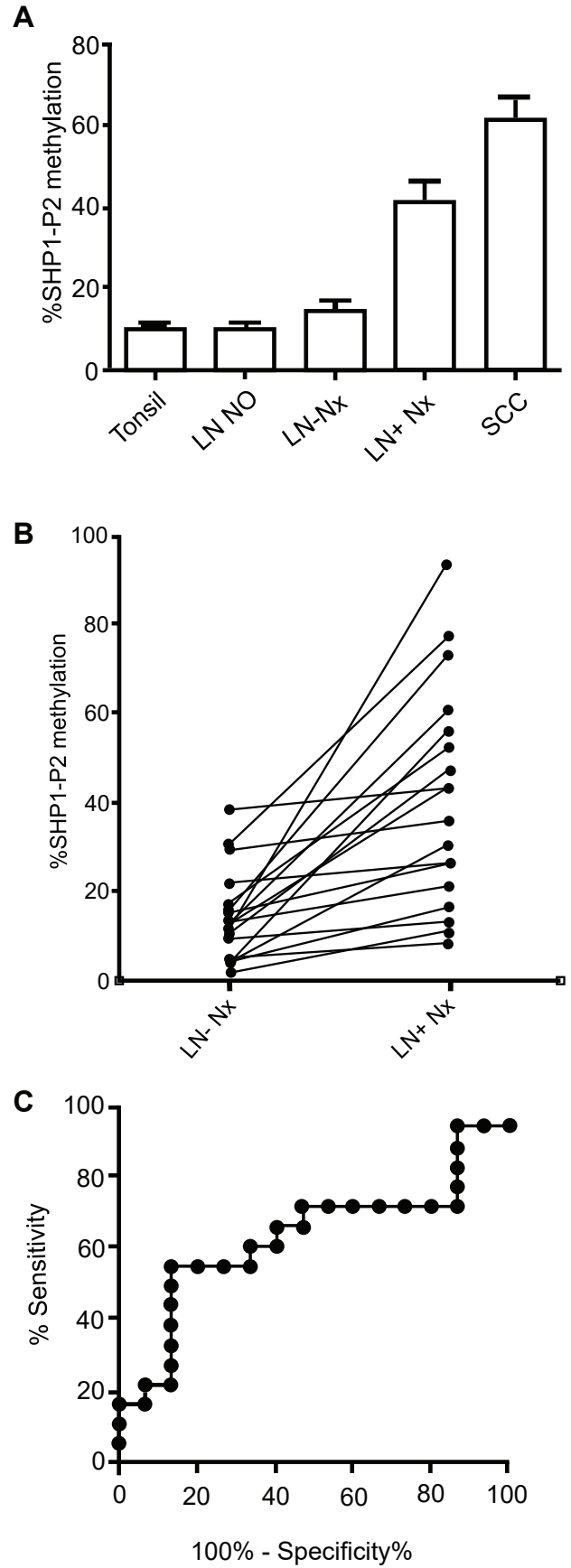

Figure 1. SHP1-P2 methylation. (A) Percentage of SHP1-P2 methylation comparisons in the 5 sample groups. (B) Comparison of LN- Nx and $\mathrm{LN}+\mathrm{Nx}$. A significant difference was observed between $\mathrm{LN}-\mathrm{Nx}$ and $\mathrm{LN}+\mathrm{Nx}(P<0.0001)$. (C) ROC curve analysis to distinguish LN N0 from LN- Nx. The curve showed AUC $=0.637$ with a sensitivity of $66.67 \%$ and a specificity of $60 \%$. AUC, area under the curve; HNSCC, squamous cell carcinoma of the head and neck; LN NO, nonmetastatic HNSCC lymph nodes; LN-Nx = lymph nodes that are histologically negative for tumor cells in patients with metastatic HNSCC; LN+ Nx, lymph nodes that are histologically positive for tumor cells in metastatic HNSCC; ROC, receiver operating characteristic; SCC, squamous cell carcinoma; SHP1-P2, Src homology region 2 domain-containing protein-tyrosine phosphatase 1 promoter 2 patients with 
Table 2. Percentage of SHP1-P2 methylation

\begin{tabular}{lcc}
\hline Group & $\mathbf{n}$ & SHP1-P2 methylation (\%, mean \pm SD) \\
\hline Tonsil & 10 & $10.27 \pm 4.05$ \\
LN N0 & 15 & $9.99 \pm 6.61$ \\
LN- Nx & 18 & $14.49 \pm 10.03$ \\
LN+ Nx & 18 & $41.01 \pm 24.51$ \\
SCC & 9 & $61.31 \pm 17.00$ \\
\hline
\end{tabular}

HNSCC, squamous cell carcinoma of the head and neck; LN NO, nonmetastatic HNSCC lymph nodes, LN- Nx, lymph nodes that are histologically negative for tumor cells in patients with metastatic HNSCC; LN- Nx, lymph nodes that are histologically positive for tumor cells in patients with metastatic HNSCC; SCC, squamous cell carcinoma; SHP1-P2, SrC homology region 2 domain-containing protein-tyrosine phosphatase 1 promoter 2

micrometastatic tumor cells or DNA, including serial section staining, immunohistochemistry, and PCR or reverse transcription-PCR of various genes [15-19]. However, the existing methods are inconclusive and do not provide satisfactory results. At present, immunohistochemistry with a cytokeratin antibody is used widely [20]. However, it is very difficult to detect only a minute HNSCC cell in the lymph node and sometimes it is not possible to see any HNSCC cell by observing just 1 section. Molecular techniques are more sensitive than immunohistochemistry, and can detect HNSCC DNA without tumor cell detection [18, 21].

DNA methylation is a common epigenetic event in cancer and plays an important role in tumor progression [22, 23]. $S H P 1-P 2$ is a tissue-specific gene. SHP1-P2 methylation is proven to be inversely correlated with expression in epithelial cells [8]. Because there is no report of SHP1-P2 mutation, $S H P 1-P 2$ methylation is considered responsible for $S H P 1-P 2$ expression. Here we observed SHP1-P2 methylation; methylated SHP1-P2 DNA is epithelial cell specific and may be used as a universal epithelial tumor marker for metastasis in lymph nodes. In our previous study of CRC, COMST PCR was used to detect SHP1-P2 methylation levels in CRC metastasis in lymph nodes without microscopically detectable cancer cells and the methylation levels were found to be higher than those in the nonmetastatic CRC lymph nodes $(P<0.001)$ [12]. The COMST PCR technique is sufficiently sensitive to detect the DNA from a single epithelial cell and has been shown to have a high precision in distinguishing epithelial cells from other cell types based on their DNA methylation level [12].

The results of this study showed that SHP1-P2 promoter methylation is significantly different in the tonsil group (organ that contains only lymphoid tissue) compared with that in microdissected HNSCC epithelia (totally epithelial cancer cells). Thus, this technique is highly sensitive for detecting HNSCC cells. The SHP1-P2 methylation level in LN N0 was the same as that in the tonsil group as the tissue types are the same. The case-matched evaluation of patients with LN+ $\mathrm{Nx}$ compared to $\mathrm{LN}-\mathrm{Nx}$ demonstrated significantly higher SHP1-P2 methylation in LN+ Nx. These results confirmed the concordant effect of HNSCC cells in the lymph nodes. Then, we assessed this marker for its applicability to HNSCC micrometastasis detection using the $\mathrm{LN}-\mathrm{Nx}$ samples as representatives. Unfortunately, we did not find a significant difference in the levels of SHP1-P2 methylation between LN N0 and $\mathrm{LN}-\mathrm{Nx}$. Therefore, this marker is far from conclusive for HNSCC micrometastasis detection, which might be attributed to the possibility that the $\mathrm{LN}-\mathrm{Nx}$ could be at a high level in normal lymphocytes with very little or no HNSCC DNA in the sample for examination. Furthermore, SHP1-P2 methylation levels were not correlated with the clinical stage or histological grade (data not shown), which is consistent with previous findings $[12,21]$.

A cutoff value of $10.14 \%$ was chosen when considering both highest levels of sensitivity and specificity level. At this suitable value, we were able to develop a useful test for distinguishing lymph nodes that are metastatic tumor positive with a sensitivity of $66.7 \%$ and specificity of $60 \%$. Therefore, we do not recommend that SHP1-P2 methylation be used for confirmation; histopathological examination should remain the criterion standard for a definitive diagnosis. Our findings also suggest the potential use of this technology as an ancillary tool for detecting micrometastatic tumors in lymph node metastases, in cases where tumor cells are not detected by routine pathological examination. The sensitivity and specificity of using the single epithelial marker for detecting HNSCC cells in lymph nodes is promising. In future work, we suggest investigating the use of SHP1-P2 methylation in combination with other biomarkers, such as long interspersed nuclear element-1 and Alu methylation [21]. Given the limited sample size, evaluation of a larger population should also be performed.

Author contributions. NK and AM substantially contributed to the conception and design of the study. SK, JW, and PR substantially acquired the data, and NK analyzed and interpreted it. NK and PR drafted the manuscript. SK, JW, and AM critically revised it. NK, SK, JW, and AM approved the final version submitted for publication and take responsibility for the statements made in the published article; NK as corresponding author takes responsibility for PR in this regard.

Acknowledgments. We thank the staff of the Department of Pathology, Faculty of Medicine, Chulalongkorn University, Bangkok, Thailand, for their assistance in collecting and providing the samples. This study was financially supported by Faculty of Dentistry, Mahidol University, Bangkok, Thailand. 
The present work was presented in part as a poster at the 41st Annual Conference of the Anatomy Association of Thailand (AAT41), 2018 May 23-25, Cha-am, Phetchaburi, Thailand. Nakarin Kitkumthorn, Somboon Keelawat, Prakasit Rattanatanyong, Apiwat Mutirangura. Evaluation of SHP1-P2 methylation as a biomarker of lymph node metastasis in head and neck squamous cell carcinoma patients [PP 37, AAT26].

Conflict of interest statement. With the exception of Prakasit Rattanatanyong, for whom the corresponding author takes responsibility, the authors have each completed an International Committee of Medical Journal Editors Uniform Disclosure Form for Potential Conflicts of Interest. None of the authors has any potential conflict of interest to disclose.

\section{References}

[1] Manikantan K, Dwivedi RC, Sayed SI, Pathak KA, Kazi R. Current concepts of surveillance and its significance in head and neck cancer. Ann R Coll Surg Engl. 2012; 93:576-82.

[2] Zhang X, Hunt JL, Shin DM, Chen ZG. Down-regulation of S100A2 in lymph node metastases of head and neck cancer. Head Neck. 2007; 29:236-43.

[3] Gu CD, Osaki T, Oyama T, Inoue M, Kodate M, Dobashi K, et al. Detection of micrometastatic tumor cells in pN0 lymph nodes of patients with completely resected nonsmall cell lung cancer: impact on recurrence and Survival. Ann Surg. 2002; 235:133-9.

[4] Coello MC, Luketich JD, Litle VR, Godfrey TE. Prognostic significance of micrometastasis in non-small-cell lung cancer. Clin Lung Cancer. 2004; 5:214-25.

[5] Imoto S, Ochiai A, Okumura C, Wada N, Hasebe T. Impact of isolated tumor cells in sentinel lymph nodes detected by immunohistochemical staining. Eur J Surg Oncol. 2006; 32:1175-9.

[6] Broglie MA, Haile SR, Stoeckli SJ. Long-term experience in sentinel node biopsy for early oral and oropharyngeal squamous cell carcinoma. Ann Surg Oncol. 2011; 18:2732-8.

[7] Rahbari NN, Bork U, Motschall E, Thorlund K, Büchler MW, Koch $\mathrm{M}$, Weitz J. Molecular detection of tumor cells in regional lymph nodes is associated with disease recurrence and poor survival in node-negative colorectal cancer: a systematic review and meta-analysis. J Clin Oncol. 2012; 30:60-70.

[8] Ruchusatsawat K, Wongpiyabovorn J, Shuangshoti S, Hirankarn N, Mutirangura A. SHP-1 promoter 2 methylation in normal epithelial tissues and demethylation in psoriasis. J Mol Med (Berl). 2006; 84:175-82.

[9] Vinayanuwattikun C, Sriuranpong V, Tanasanvimon S, Chantranuwat P, Mutirangura A. Epithelial-specific methylation marker: a potential plasma biomarker in advanced non-small cell lung cancer. J Thorac Oncol. 2011; 6:1818-25.

[10] Vinayanuwattikun C, Mingmalairak S, Jittapiromsak N, Thaipisuttikul I, Sriuranpong V, Mutirangura A, Shuangshoti S. SHP-1 promoter 2 methylation in cerebrospinal fluid for diagnosis of leptomeningeal epithelial-derived malignancy (carcinomatous meningitis). J Neurooncol. 2016; 129:395-403.

[11] Vinayanuwattikun C, Chantranuwat P, Sriuranpong V, Mutirangura A. The role of SHP-1 promoter 2 hypermethylation detection of lymph node micrometastasis in resectable stage I non-small cell lung cancer as a prognostic marker of disease recurrence. Int J Clin Oncol. 2014; 19:586-92.

[12] Rattanatanyong P, Keelawat S, Kitkumthorn N, Mutirangura A. Epithelial-specific SHP1-P2 methylation - a novel universal tumor marker for detection of colorectal cancer lymph node metastasis. Asian Pac J Cancer Prev. 2016; 17:4117-23.

[13] Kitkumthorn N, Mutirangura A. LINE-1 methylation difference between ameloblastoma and keratocystic odontogenic tumor. Oral Dis. 2010; 16:286-91.

[14] Patel AN, Mehnert JM, Kim S. Treatment of recurrent metastatic head and neck cancer: focus on cetuximab. Clin Med Insights Ear Nose Throat. 2012; 5:1-16.

[15] Tsavellas G, Patel H, Allen-Mersh TG. Detection and clinical significance of occult tumour cells in colorectal cancer. Br J Surg. 2001; 88:1307-20.

[16] Riethdorf S, Wikman H, Pantel K. Review: Biological relevance of disseminated tumor cells in cancer patients. Int J Cancer. 2008; 123:1991-2006

[17] Wada N, Imoto S. Clinical evidence of breast cancer micrometastasis in the era of sentinel node biopsy. Int J Clin Oncol. 2008; 13:24-32.

[18] Shimizu Y, Takeuchi H, Sakakura Y, Saikawa Y, Nakahara T, Mukai $\mathrm{M}$, et al. Molecular detection of sentinel node micrometastases in patients with clinical N0 gastric carcinoma with real-time multiplex reverse transcription-polymerase chain reaction assay. Ann Surg Oncol. 2012; 19:469-77.

[19] Patel V, Martin D, Malhotra R, Marsh CA, Doçi CL, Veenstra TD, et al. DSG3 as a biomarker for the ultrasensitive detection of occult lymph node metastasis in oral cancer using nanostructured immunoarrays. Oral Oncol. 2013; 49:93-101.

[20] Barrera JE, Miller ME, Said S, Jafek BW, Campana JP, Shroyer KR. Detection of occult cervical micrometastases in patients with head and neck squamous cell cancer. Laryngoscope. 2003; 113:892-6.

[21] Kitkumthorn N, Keelawat S, Rattanatanyong P, Mutirangura A. LINE-1 and Alu methylation patterns in lymph node metastases of head and neck cancers. Asian Pac J Cancer Prev. 2012; 13:4469-75.

[22] Kitkumthorn N, Mutirangura A. Long interspersed nuclear element-1 hypomethylation in cancer: biology and clinical applications. Clinical Epigenetics. 2011; 2:315.

[23] Chujan S, Kitkumthorn N, Siriangkul S, Mutirangura A. CCNA1 promoter methylation: a potential marker for grading Papanicolaou smear cervical squamous intraepithelial lesions. Asian Pac J Cancer Prev. 2014; 15:7971-5. 